\title{
Physiotherapy management of patients with Coronary Artery Disease: a Report on current practice in South Africa
}

\begin{abstract}
Coronary artery disease (CAD) is a worldwide health problem with an increased prevalence in sub-Saharan Africa. Physiotherapists internationally are involved in the care of these patients from the acute stage following a cardiac event until phase III cardiac rehabilitation is completed. The purpose of this study was to determine the current physiotherapy management of patients with CAD in South Africa. An observational crosssectional study was conducted over two months with a questionnaire that was sent to the government and private health care sectors. Results showed that more cardiopulmonary physiotherapists provided care (62\%) than those who didn't (38\%). Care was mostly provided in a hospital setting (81\%) and out- patient phase III cardiac rehabilitation was lacking (11\%). In-hospital physiotherapy treatment was mostly provided once daily. Deep breathing exercises (99\%), circulatory exercises (95\%) and manual chest clearance techniques (88\%) were mostly used during physiotherapy. Evidence based practice was consistent regarding early mobilization but was inconsistent with regards to the use of manual chest clearance techniques.
\end{abstract}

\section{KEY WORDS: CORONARY ARTERY DISEASE, PHYSIOTHERAPY, CURRENT PRACTICE.}

\section{INTRODUCTION}

Coronary artery disease (CAD) is a health problem worldwide. It is the leading cause of death internationally and accounts for a third of deaths globally (Mackay and Menash 2004). Mortality from CAD in developed countries has declined due to improved prevention strategies, diagnosis and treatment (Mackay and Menash 2004). CAD was previously considered rare in subSaharan Africa but due to epidemiological transition (economic development, industrialisation and urbanisation) this is no longer the case (Mensah 2008). Epidemiological transition results in the shift of major causes of death from infectious diseases and nutritional defi-

\footnotetext{
Correspondence to:

Ronel Roos

Cardiopulmonary lecturer

Department of Physiotherapy

School of Therapeutic Sciences

University of the Witwatersrand

7 York Road

Parktown 2193

E-mail: ronel.roos@wits.ac.za
}

ciencies to a predominance of chronic lifestyle diseases such as CAD. South Africa is said to be in the second and third stage of epidemiological transition as the prevalence of risk factors for $\mathrm{CAD}$ and the disease itself are increasing in a more compressed time frame when compared to developed countries (Mensah 2008; Steyn et al 2005). In the Interheart Africa study the researchers demonstrated that hypertension, abdominal obesity as reflected by waist-hip ratio, current/former smoking status and stress levels were stronger predictors for acute myocardial infarction in the Africa study group compared to the global Interheart study (Steyn et al 2005). The presence of lifestyle risk factors for CAD is therefore clearly evident. Another component to be aware of specifically when looking at CAD in the African context is the effect that the human immunodeficiency virus (HIV) has on the prevalence of CAD. The risk for CAD is higher in people infected with HIV and occurs at an earlier age (Currier et al 2003). Due to all these factors it is no wonder that CAD is projected to be one of the leading causes of death in developing countries by 2030 (Abegunde et al 2007).

Internationally, physiotherapists treat patients with CAD in the acute stage following a coronary event and/ or following coronary artery bypass graft (CABG) surgery. These patients are then subsequently followed up as outpatients during cardiac rehabilitation in order to improve function and quality of life and to delay the occurrence of subsequent coronary events (Piotrowicz and Wolszakiewicz 2008; Martin 2007; Tucker et al 1996). The role of the South African physiotherapist in the care and rehabilitation of patients with $\mathrm{CAD}$ has not been investigated to date. It is not clear what treatment interventions South African physiotherapists use in their management of such patients. The aims of this study were to establish a) how many physiotherapists who work in the cardiopulmonary field of physiotherapy are involved in the care and rehabilitation of patients with CAD; b) the current physiotherapy interventions that are used in the management of patients with CAD; c) the clinical setting (acute care or outpatient care) in which patients 
with CAD regularly receive physiotherapy interventions; d) the frequency of physiotherapy interventions and follow-up of patients with CAD and e) the reasons why physiotherapists working in a cardiopulmonary setting may not be involved in rehabilitation of patients with CAD.

\section{METHODOLOGY}

An observational cross-sectional study was conducted. A questionnaire was developed following a literature review of chest clearance and rehabilitation methods used in the management of patients with CAD. A review of cardiac procedures performed in individuals diagnosed with CAD was also done. The questionnaire was developed in English as it is the language of medical science (nationally and internationally). The questionnaire was discussed with a peer group to verify its contents, to establish the time required to complete the questionnaire, to establish if the questions addressed the aims of the study and if all questions were understandable to the participant. Minor changes were made to the questionnaire. The questionnaire, informed consent form and information sheet were mailed to the physiotherapy heads of department (HOD) of government hospitals (identified through the Department of Health webpage) throughout South Africa that have intensive care units. The HOD was asked to invite his/her staff who worked in a cardiopulmonary setting to participate in the study. The questionnaires were then either faxed back to the researcher or mailed back in the enclosed pre-paid addressed envelope.

Secondly, the South African Society of Physiotherapy (SASP) directory was reviewed to determine which private physiotherapy practices provided cardiopulmonary care to patients. These practices were contacted telephonically to determine if they were willing to participate in the study. On agreement each participant's e-mail address was obtained and the questionnaire, informed consent form and information sheet were then circulated electronically to them. Thirdly, an information letter of the study was sent to the chairperson of the Cardiopulmonary Rehabilitation Special
Interest Group (CPRG) of the SASP. The letter requested that the researcher be permitted access to the CPRG members' e-mail addresses in an attempt to circulate the questionnaire to them. All documents were then e-mailed to the CPRG members of the SASP. The private practitioners and CPRG members had the option of returning the questionnaire via e-mail as an attachment or to fax it back to the researcher. All study participants had a two month period (June \& July 2009) in which to return the questionnaire to the researcher and reminder strategies (telephone calls and e-mail) were used to remind participants of the submission deadline. Permission to conduct this study was obtained from the University of the Witwatersrand Human Research Ethics committee.

Figure 1: Interventions used in management of CAD patients.

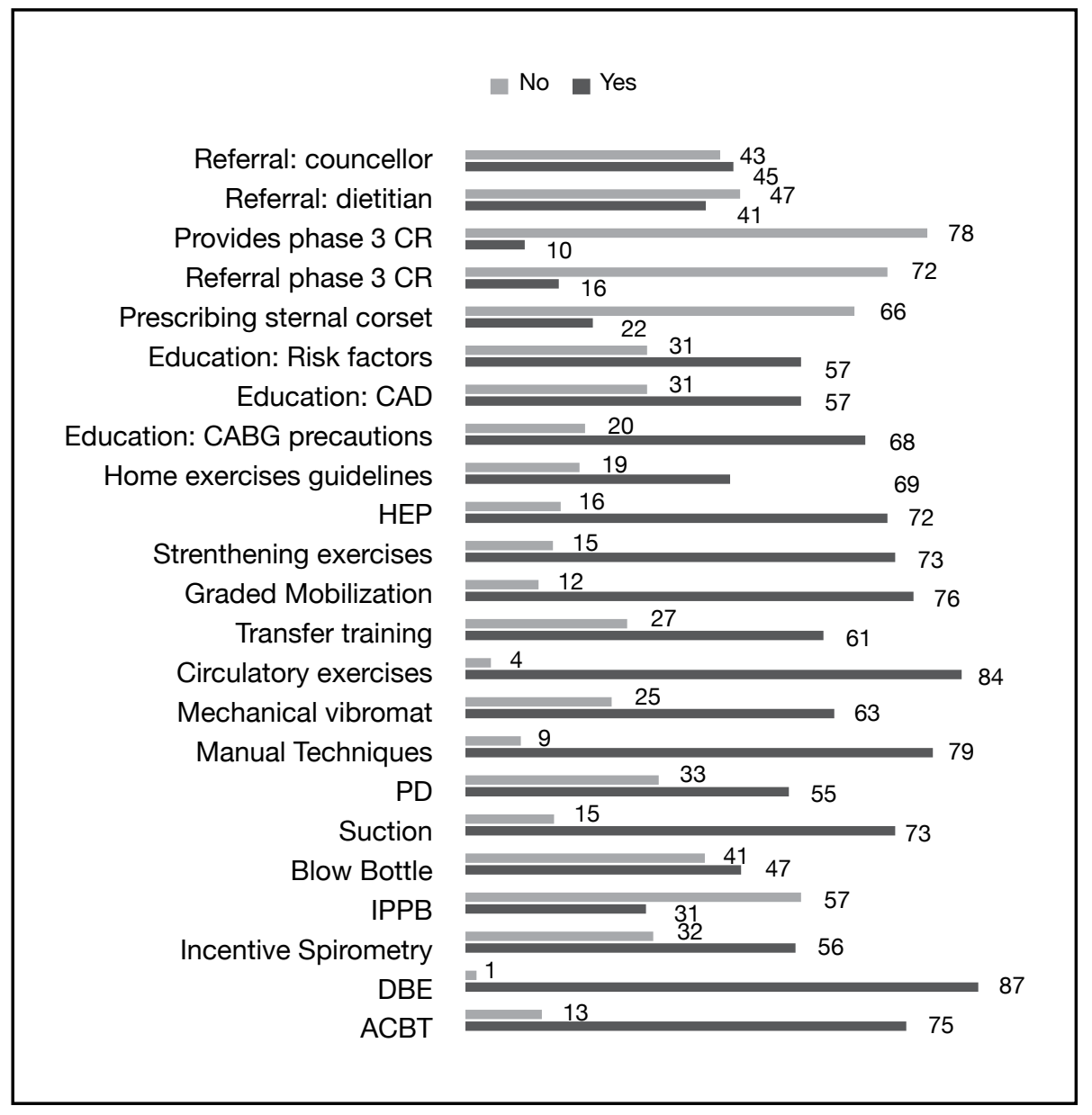

Data expressed as number of physiotherapists using interventions. CR, cardiac rehabilitation; CAD, coronary artery disease; CABG, coronary artery bypass graft; HEP, home exercise programme; PD, postural drainage; IPPB, intermittent positive pressure breathing; DBE, deep breathing exercises; ACBT, active cycle of breathing technique.
Descriptive data analysis was used to describe the data.

\section{RESULTS}

The questionnaire was mailed to 50 regional and tertiary government institutions and 137 electronic questionnaires were circulated. A total of 187 questionnaires were sent out and 142 were returned (76\%). Results showed that $62 \%$ of the physiotherapists provided care to patients with CAD (50 government physiotherapists and 38 private practitioners) of the $38 \%$ who did not treat patients with $\mathrm{CAD}, 38$ were government physiotherapists and 16 private practitioners.

Figure 1 outlines the interventions used by physiotherapists when treating patients with CAD. It is important to note 
that deep breathing exercises, circulatory exercises, manual techniques and postural drainage, graded mobilisation and education components (home exercise program, exercise guidelines and $\mathrm{CABG}$ precautions) were commonly included as treatment modalities. Intermittent positive pressure breathing (IPPB) was not commonly used as a chest clearance technique. Only 16 physiotherapists referred patients to a phase III out-patient cardiac rehabilitation programme and ten cardiopulmonary physiotherapists offered such a programme at the hospital

Table 1: Frequency of physiotherapy interventions

\begin{tabular}{|l|l|}
\hline In hospital & Number \\
\hline Once daily & 52 \\
\hline Twice daily & 42 \\
\hline Three times daily & 6 \\
\hline Do not provide PT in hospital & 4 \\
\hline Outpatient setting & \\
\hline Two/three times weekly & 3 \\
\hline Once weekly & 17 \\
\hline Once in two weeks & 6 \\
\hline Once in a month & 5 \\
\hline Do not provide PT in outpatient setting & 56 \\
\hline Depends on patient & 1 \\
\hline
\end{tabular}

Figure 2: Clinical settings and scenarios.

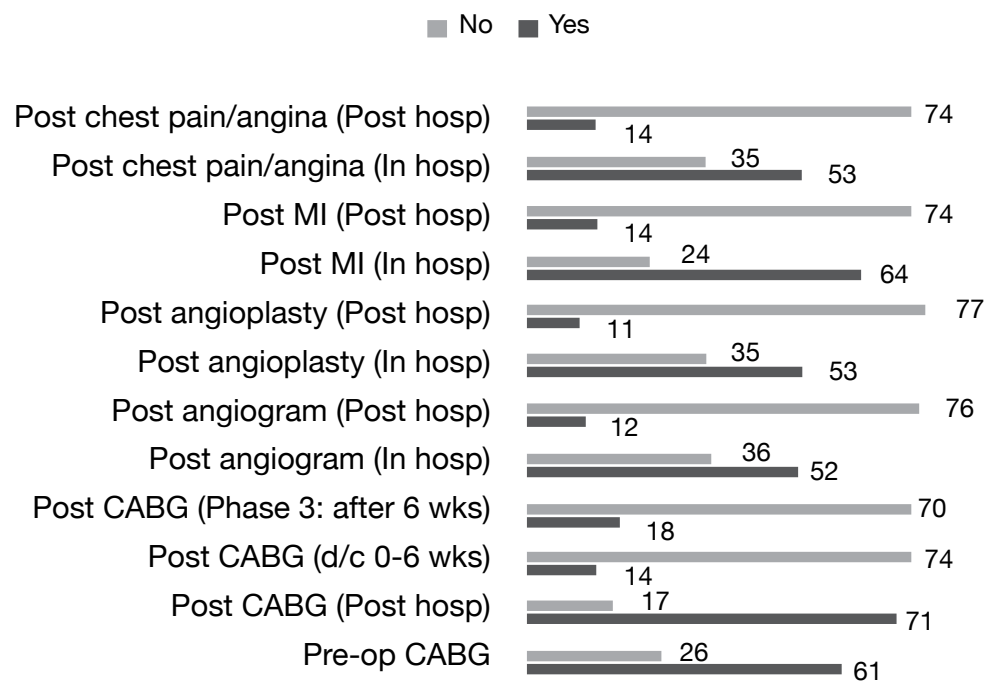

Data expressed as number of physiotherapist working in clinical settings/ scenarios. MI, myocardial infarction; CABG, coronary artery bypass graft reason for referral of these patients to physiotherapy is unknown. Few physiotherapists continued with patient care following discharge from the hospital.

\section{REASONS FOR NOT PROVIDING REHA- BILITATION TO PATIENTS WITH CAD}

The most common reasons listed for not providing rehabilitation care to patients with CAD in the private sector included a lack of equipment, space and time. It was mentioned that patients often received information from nursing staff members concerning living a healthy lifestyle and from dieticians concerning an appropriate diet. Some private practitioners did provide outpatient rehabilitation but this was discontinued mostly due to poor patient adherence or changes in doctors' referral patterns. It was also noted that patients often lived far away from the specialised center where their coronary event was managed. Follow-up of these patients was therefore not possible. In the public sector these patients were often not referred to the physiotherapy department or they were transferred to a more specialised hospital. It was also noted that intervention in these patients depended on the patient's clinical condition e.g. the presence of post operative complications or if they were medically stable.

\section{DISCUSSION}

Coronary artery disease is projected to be the leading cause of death in developing countries by 2030 (Abegunde 2007). This study investigated the current level of involvement of cardiopulmonary physiotherapists in the management of patients with CAD in South Africa.

It was encouraging to note that more cardiopulmonary physiotherapists provided care to patients living with CAD than those who did not. Primary prevention strategies such as education concerning living a healthy lifestyle and secondary management for reoccurrence of a cardiac event such as cardiac rehabilitation are important components in attempting to decrease the burden of CAD (Mensah 2008; Piotrowicz and Wolszakiewicz 2008). Education consisting of postoperative precautions of CABG (wound care and temporary restrictions in physical 
activity), exercise guidelines (including frequency, intensity, time and type of exercises and red flags), general CAD pathophysiology and risk factor modification was done by physiotherapists in the acute care setting. This is commendable because the motivation of patients with CAD to be active appears to be greatest in the early post-discharge period (Reid et al, 2006). Physical activity levels in patients are reported to decline two to six months following hospital discharge after a CAD event (Reid et al, 2006). Interventions to assist with increasing physical activity levels during this period, such as phase III cardiac rehabilitation, should be encouraged (Reid et al, 2006). The small number of physiotherapists involved with phase III cardiac rehabilitation in this study is also a cause for concern. It is unclear if patients received cardiac rehabilitation from other health care professionals such as nursing staff or biokineticists.

The questionnaire revealed that deep breathing exercises (DBE) (87) were the most common chest clearance technique used by physiotherapists when treating patients with CAD followed by manual techniques (79), active cycle of breathing technique (ACBT) (75), postural drainage (55), incentive spirometry (IS) (56) and blow-bottle (47). The use of DBE is supported by Tucker et al (1996) and Martin (2007) as techniques commonly used by physiotherapists in the management of patients undergoing CABG surgery in Australia and the United Kingdom. There is however conflicting evidence in the literature concerning the effectiveness of $\mathrm{DBE}$ following CABG and for the purpose of this discussion the treatment technique will be divided into positive pressure related and non-pressure related deep breathing exercises.

Westerdahl et al (2005) conducted a randomised controlled trial (level $1 b$ ) in patients following CABG and found that implementing DBE with a fluctuating positive pressure device e.g. blow-bottle improved the patients' pulmonary function. CT-scans of these patients' chests revealed less areas of atelectasis in the intervention group. It is important to note that the DBE in this study was performed in a sitting position. It is well known that an upright position is more adventitious compared to the supine position when attempting to improve lung volumes in a cardiopulmonary patient (Pryor and Prasad 2008; Frownfelter and Dean 2006). Intermittent positive pressure breathing (IPPB) was used by 31 physiotherapists in the current study. Romanini et al (2007) reported that IPPB was effective in managing hypoxemia in CABG patients in the first few days following surgery. However, Westerdahl et al (2001) reported that DBE performed with a blow bottle device was more effective in preventing a decline in lung function in patients after CABG than DBE alone or IPPB. Brasher et al (2003) on the other hand showed that if DBE was removed from an early mobilisation program for patients after CABG there was no significant difference between the control and intervention groups relating to the patients' lung function results. In this and Westerdahl's studies the patients were mobilised out of bed on day one post surgery but the positioning and repetitions during DBE were different. In Westerdahl's study (2005) all DBE were done in sitting and the repetitions of DBE per hour were greater compared to the latter study where participants were positioned in supine or sitting during DBE and performed fewer repetitions.

With reference to non-pressure related DBE, the effect of IS was compared to other prophylactic physiotherapy techniques on postoperative pulmonary complications (PPC) following CABG in a Cochrane review (Freitas et al 2007) (level 1a evidence). A total of four randomised controlled trials met the inclusion criteria for this review. The reviewers found no differences in PPC when using IS or a pressure device such as IPPB for patients after CABG surgery. Worse pulmonary function and arterial oxygen levels were reported with the use of IS when compared with pressure devices (Freitas et al, 2007). Even though the evidence is contradictory in nature regarding the use of chest clearance techniques such as DBE, IS, blow-bottle and IPPB post-operatively, patients who undergo $\mathrm{CABG}$ surgery have significant pulmonary dysfunction four days postoperatively which can persist up to four months following surgery (Westerdahl et al, 2003). Intervention strategies used by physiotherapists for the improvement of pulmonary function following $\mathrm{CABG}$ is therefore still justified.

Manual techniques were frequently used by physiotherapists in the current study. A literature search in Pubmed, Cochrane Database for Systematic Reviews and Scopus however found no evidence to support the use of this treatment modality in the care of patients with CAD. Postural drainage (PD) was also commonly used. Head-down PD is considered a relative contraindication in patients with severe cardiovascular disease (Hough 1997). A short-term 30 degree head-down PD session in healthy adults resulted in decreased heart rate, mean arterial blood pressure and diastolic duration (Naylor 2005). In healthy adults these changes may have no serious consequences but in individuals with known $\mathrm{CAD}$ and reduced cardiac reserve it could result in significant side effects. Naylor et al (2006) conducted a quasi-experimental study to evaluate the cardiovascular (CVS) and respiratory responses to modified and head-down PD positioning in patients with severe heart disease (mean ejection fraction $23 \%$ SD 7). The researchers found no significant differences in respiratory responses in participants when positioned in a modified or headdown PD position. There was a significant difference in CVS responses when the two positions were compared and modified PD was better tolerated. It is therefore advisable to use a modified PD position in patients with CAD if indicated. Close monitoring of the patient's response while in the specific position should be maintained.

Activity based interventions such as circulatory exercises and/or graded mobilisation were commonly used by physiotherapists in this study. These interventions are supported by evidence in the literature. Early mobilisation in hospital is encouraged following $\mathrm{CABG}$ surgery and acute coronary events if patients are medically stable (Hirschhorn et al 2008; Pryor and Prasad 2008). In the majority of articles mentioned thus far the patients were mobilised out of bed on day one post surgery and mobili- 
sation progressed gradually. Hirschhorn et al (2008) illustrated with a randomised controlled trial (level $1 \mathrm{~b}$ ) that a moderate intensity (3-4 on modified Borg scale) walking program following $\mathrm{CABG}$ was safe and effective in increasing walking distance at discharge.

In this study, patients with CAD were most commonly seen by physiotherapists once or twice daily while in hospital. This finding is similar to the frequency of treatment provided by physiotherapists working in the United Kingdom when treating patients post cardiac surgery (Martin 2007). When reviewing treatment frequency Van der Peijl et al (2004) reported no difference in hospital length of stay or postoperative complications between patients seen once or twice daily by physiotherapists after cardiac surgery. They did note that the twice daily group reached functional milestones sooner and was more satisfied with the physiotherapy service.

A group of patients that was not so frequently managed by physiotherapists was that undergoing angiogram and angioplasty interventions. It is well known that these patients are less physically active than patients who have had CABG (Reid et al 2006). The role of education in the form of risk factor modification and the importance of exercise is essential for such patients as the rate of angiography interventions is increasing (Reid et al 2006).

\section{CONCLUSION}

The study confirmed that most physiotherapists working in the cardiopulmonary field in South Africa are involved in the management of patients with CAD during hospitalisation. However the use of evidence based interventions in the clinical care of patients with CAD was inconsistent. There is currently limited involvement of physiotherapists in outpatient cardiac rehabilitation in South Africa. Considering the potential burden of this disease on the economy of South Africa, the focus of physiotherapists should be on prevention of risk factors such as hypertension through education and exercise as well as implementation of programmes (cardiac rehabilitation) for the long-term management of individuals diagnosed with CAD.
Physiotherapy practice in this arena could be enhanced by evidenced-based workshops to clinicians, improved communication between professionals [referrals], creating a data base of healthcare professionals providing phase three cardiac rehabilitation and outpatient followup of patients to enhance patient care.

\section{ACKNOWLEDGEMENTS}

The researcher would like to thank all the government and private sector physiotherapists who participated in this study.

\section{REFERENCES}

Abegunde DO, Mathers CD, Adam T, Ortegon M, Strong K 2007 The burden and cost of chronic diseases in low-income and middle-income countries. Lancet 370: 1929-1938.

Brasher PA, McClelland KH, Denehy L, Story I 2003 Does removal of deep breathing exercises from a physiotherapy program including preoperative education and early mobilization after cardiac surgery alter patient outcomes? Australian Journal of Physiotherapy 49: 165-173.

Currier JS, Taylor A, Boyd F, Dezii CM, Kawabata H, Burtcel B, Maa JF, Hodder S 2003 Coronary Heart Disease in HIV Infected Individuals. Journal of Acquired Immune Deficiency Syndrome 33: 506-512.

Freitas ERFS, Soares B, Cardoso JR, Atallah AN 2007 Incentive spirometry for preventing pulmonary complications after coronary artery bypass graft. Cochrane Database of Systematic Reviews, Issue 3. Art No.:CD004466.DOI: 10.1002/14651858.OD004466.pub2.

Frownfelter D, Dean E 2006 Cardiovascular and pulmonary physical therapy: evidence and practice, $4^{\text {th }}$ edn. pp. $307-321$. Elsevier Publishers, Toronto.

Hirschhorn AD, Richards D, Mungovan SF, Morris NR, Adams L 2008 Supervised moderate intensity exercise improves distance walked at hospital discharge following coronary artery bypass graft surgery: a randomised controlled trial. Heart, Lung and Circulation 17: 129-138.

Hough A 1997 Physiotherapy in Respiratory Care, 2edn.pp. 132-133. StanleyThornes(Publishers)Ltd.

Mackay J \& Menash GA 2004 The atlas of heart disease and stroke. Retrieved 21/07/2009

http://www.who.int/cardiovascular_diseases/ resources/atlas/en/index.html.

Martin AC 2007 Current physiotherapy practice for post-operative cardiac patients. Journal of the Association of Chartered Physiotherapists in Respiratory Care 39: 27-31.

Mensah GA 2008 Ischaemic heart disease in Africa. Heart 94: 836-843.
Naylor JM, Chow CM, Mclean AS, Heard RC, Avolio A 2005 Cardiovascular responses to short-term head-down positioning in healthy young and older adults. Physiotherapy Research International 10(1): 32-47.

Naylor Jm, Mclean A, chow CM, Heard R, Ting I, Avolio A 2006 a modified postural drainage position produces less cardiovascular stress than a head-down position in patients with severe heart disease: A quasi-experimental study. Australian Journal of Physiotherapy 52: 201-209.

Piotrowicz R, Wolszakiewicz J 2008 Cardiac rehabilitation following myocardial infarction. Cardiology Journal 15: 481-487.

Pryor JA, Prasad SA 2008 Physiotherapy for respiratory and cardiac problems; adults and paediatrics, $3^{\text {rd }}$ edn. pp 115-130, 424-428,470-494. Churchill Livingstone, Edinburgh.

Reid RD, Morrin LI, Pipe AL, Dafoe WA, Higginson LAJ, Wielgosz AT, McDonald PW, Plotnikoff RC, Courneya KS, Oldridge NB, Beaton LJ, Papadakis S, D'Angelo MES, Tulloch HE, Blanchard CM 2006 Determinants of physical activity after hospitalization for coronary artery disease: the Tracking exercise after cardiac hospitalization (TEACH) study. European Journal of Cardiovascular Prevention and Rehabilitation 13: 529-537.

Romanini W, Muller AP, De Carvalho KAJ, Olandosi M, Faria-Neto JR, Mendes FL, Sardetto EA, Da Costa FDA, Guarita-Souza LC (2007) The effect of intermittent positive pressure and incentive spirometry in the postoperative management of myocardial revascularization. Arquivos Brasilieros de Cardiology 89(2): 94-99.

Steyn K, Sliwa K, Hawken S, Commerford P, Onen C, Damasceno A, Ounput S, Yusuf S 2005 Risk factors associated with myocardial infarction in Africa. Circulation 112: 3554-3561.

Tucker B, Jenkins S, Davies K, McGram R, Waddell J, King R, Kirby V, Lloyd C 1996 The physiotherapy management of patients undergoing coronary artery surgery: A questionnaire survey. Australian Journal of Physiotherapy 42(2): 129-137.

Van der Peijl ID, Vlieland TPMV, Versteegh MIM, Lok JJ, Munneke M, Dion RAE 2004 Exercise therapy following CABG: A randomized comparison of a high and low frequency exercise therapy program. Annals of Thoracic Surgery 77: 1535-1541.

Westerdahl E, Lindmark B, Almgren SO, Tenling A 2001 Chest physiotherapy after coronary artery bypass surgery- a comparison of three different deep breathing techniques. Journal of Rehabilitation Medicine 33: 79-84.

Westerdahl E, Lindmark B, Bryngelsson I, Tenling A 2003 Pulmonary function four months after coronary artery bypass graft surgery. Respiratory Medicine 97(4): 317-322.

Westerdahl E, Lindmark B, Eriksson T, Friberg O, Hedenstierna G, Tenling A 2005 Deep-breathing exercises reduce atelectasis and improve pulmonary function after coronary artery bypass surgery. Chest 128; 3482-3488. 